\title{
A scoping review identifies multiple emerging knowledge synthesis methods, but few studies operationalise the method
}

\author{
Andrea C. Tricco, Charlene Soobiah, Jesmin Antony, Elise Cogo, \\ Heather MacDonald, Erin Lillie, Judy Tran, Jennifer D’Souza, \\ Wing Hui, Laure Perrier, Vivian Welch, Tanya Horsley, Sharon E. \\ Straus, Monika Kastner
}

Version Post-print/Accepted Manuscript

Citation Tricco AC, Soobiah C, Antony J, Cogo E, MacDonald H, Lillie E, Tran (published version) J, D’Souza J, Hui W, Perrier L, Welch V, Horsley T, Straus SE, Kastner $\mathrm{M}, \mathrm{A}$ scoping review identifies multiple emerging knowledge synthesis methods, but few studies operationalise the method, Journal of Clinical Epidemiology (2016), doi: 10.1016/j.jclinepi.2015.08.030.

Copyright/License

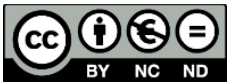
This work is licensed under the Creative Commons Attribution-NonCommercial-NoDerivatives 4.0 International License. To view a copy of this license, visit http://creativecommons.org/licenses/by-nc-nd/4.0/.

\section{How to cite TSpace items}

Always cite the published version, so the author(s) will receive recognition through services that track citation counts, e.g. Scopus. If you need to cite the page number of the author manuscript from TSpace because you cannot access the published version, then cite the TSpace version in addition to the published version using the permanent URI (handle) found on the record page.

This article was made openly accessible by $U$ of $T$ Faculty. Please tell us how this access benefits you. Your story matters. 


\section{Accepted Manuscript}

A scoping review identifies multiple emerging knowledge synthesis methods, but few studies operationalise the method

Andrea C. Tricco, Charlene Soobiah, Jesmin Antony, Elise Cogo, Heather MacDonald, Erin Lillie, Judy Tran, Jennifer D’Souza, Wing Hui, Laure Perrier, Vivian Welch, Tanya Horsley, Sharon E. Straus, Monika Kastner

PII: S0895-4356(16)00098-6

DOI: 10.1016/j.jclinepi.2015.08.030

Reference: JCE 9080

To appear in: Journal of Clinical Epidemiology

Received Date: 19 February 2015

Revised Date: 5 June 2015

Accepted Date: 7 August 2015

Please cite this article as: Tricco AC, Soobiah C, Antony J, Cogo E, MacDonald H, Lillie E, Tran J, D'Souza J, Hui W, Perrier L, Welch V, Horsley T, Straus SE, Kastner M, A scoping review identifies multiple emerging knowledge synthesis methods, but few studies operationalise the method, Journal of Clinical Epidemiology (2016), doi: 10.1016/j.jclinepi.2015.08.030.

This is a PDF file of an unedited manuscript that has been accepted for publication. As a service to our customers we are providing this early version of the manuscript. The manuscript will undergo copyediting, typesetting, and review of the resulting proof before it is published in its final form. Please note that during the production process errors may be discovered which could affect the content, and all legal disclaimers that apply to the journal pertain. 
3 Andrea C. Tricco ${ }^{\mathrm{a}, \mathrm{b}}$ email: TriccoA@smh.ca

4 Charlene Soobiah ${ }^{\mathrm{a}, \mathrm{c}}$ email: SoobiahC@ @mh.ca

5 Jesmin Antony ${ }^{\mathrm{a}} \quad$ email: AntonyJ@smh.ca

6 Elise $\mathrm{Cogo}^{\mathrm{a}}$

$7 \quad$ Heather MacDonald ${ }^{\mathrm{a}}$

8 Erin Lillie $^{\mathrm{a}}$

9 Judy $\operatorname{Tran}^{\mathrm{a}}$

10 Jennifer D'Souza ${ }^{a}$

11 Wing Hui ${ }^{\mathrm{a}}$

12 Laure Perrier $^{\mathrm{c}}$

13 Vivian Welch ${ }^{\mathrm{d}}$

14 Tanya Horsley ${ }^{\mathrm{e}}$

15 Sharon E. Straus ${ }^{\mathrm{a}, \text { f* }^{*}}$

16 Monika Kastner ${ }^{\mathrm{a}, \mathrm{b}}$ email: CogoE@smh.ca

email: MacDonaldH@smh.ca

email: LillieE@smh.ca

email: Judy.Tran@mail.utoronto.ca

email: Jennifer.Dsouza@utoronto.ca

email: Wing.hui @mail.utoronto.ca

email: 1.perrier@mail.utoronto.ca

email: Vivian.Welch@uottawa.ca

email: THorsley@ royalcollege.ca

email: Sharon.Straus@utoronto.ca

email: Monika.Kastner@utoronto.ca

${ }^{a}$ Li Ka Shing Knowledge Institute of St. Michael’s Hospital, 209 Victoria Street, Toronto,

Ontario, Canada, M5B 1W8

${ }^{\mathrm{b}}$ Epidemiology Division, Dalla Lana School of Public Health, University of Toronto, Health

Sciences Building, 155 College Street, 6th floor, Toronto, Ontario, Canada, M5T 3M7

$21{ }^{\mathrm{c}}$ Institute of Health Policy, Management and Evaluation, University of Toronto, Health Sciences

22 Building, 155 College Street, Suite 425, Toronto, Ontario, Canada, M5T 3M6

23 dibruyère Research Institute, 85 Primrose Avenue, Ottawa, Ontario, Canada, K1R 6M1 
$24{ }^{\mathrm{e}}$ Research Unit, Royal College of Physicians and Surgeons of Canada, 774 Echo Drive,

25 Ottawa, Ontario, Canada, K1S 5N8

$26{ }^{\mathrm{f}}$ Department of Geriatric Medicine, Faculty of Medicine, University of Toronto, 27 King's

27 College Circle, Toronto, Ontario, Canada, M5S 1A1

$28 *$ Corresponding Author:

29 Sharon E. Straus, MSc, MD

30 Director, Knowledge Translation program

31 Li Ka Shing Knowledge Institute of St. Michael's Hospital

32209 Victoria Street, East Building, Room 716, Toronto, Ontario, Canada, M5B 1W8

33 Phone: +1-416-864-3068, fax: +1-416-864-5805, e-mail: Sharon.Straus@utoronto.ca 34 


\section{ABSTRACT}

Objective: To systematically identify, define, and classify emerging knowledge synthesis methods through a scoping review.

Study design and setting: MEDLINE, CINAHL, Embase, PsycINFO, the Cochrane Methodology Register, the Cochrane Database of Systematic Reviews, Social Sciences Abstracts, LISA, Philosopher's Index, and ERIC were searched to identify papers reporting emerging knowledge synthesis methods across the disciplines of health, education, sociology, and philosophy. Two reviewers independently selected studies and abstracted data for each article.

Results: In total, 409 articles reporting on 25 knowledge synthesis methods were included after screening of 17,962 titles and abstracts and 1,010 potentially relevant full-text papers. The majority of the included articles were an application of the method (83.9\%); only $3.7 \%$ were seminal articles that fully described the method (ie, operationalised the steps). Most of the included articles were published after 2005. The methods were most commonly used across the fields of nursing, health care science and services, and health policy.

Conclusion: We found a lack of guidance on how to select a knowledge synthesis method. We propose convening an international group of leaders in the knowledge synthesis field to help clarify emerging approaches to knowledge synthesis.

Word count: 188 (abstract), 2710 (main text), 3 figures, 3 tables, 6 appendices.

Key Words: systematic review, knowledge synthesis, realist review, meta-ethnography, metanarrative, meta-synthesis

Running Title: Scoping review of knowledge synthesis methods 


\section{What is new}

\section{Key findings}

We found that most emerging knowledge synthesis methods were published after 2005, that most focused on nursing, health care science and services, and health policy fields, and that most represented an application of the method (83.9\%) as opposed to being seminal articles describing the method in detail (ie, operationalising the steps; $3.7 \%$ ).

\section{What this adds to what is known}

This is the first study to systematically characterise the features of novel knowledge synthesis methods.

\section{What is the implication}

We found a lack of guidance on how to select a knowledge synthesis method.

\section{What should change now}

We propose convening an international group of leaders in the field to help clarify emerging approaches to knowledge synthesis. 


\section{Introduction}

The notion of synthesising literature is not new [1]. Examples of knowledge synthesis in the field of philosophy date back to the 12th century, and statistical techniques for synthesising literature were common practice in astronomy in the 17th century. One of the first examples of a rudimentary meta-analytic approach in the health-related literature, in which studies on typhoid vaccination were pooled, was published in 1904 [2]. Statisticians working in the disciplines of education, social sciences, and psychology began advancing modern meta-analytic techniques in the 1970s [3-7].

Empirical methods for conducting systematic reviews and meta-analyses of medical interventions have been formally established through over four decades of work by many organisations internationally, in particular the Cochrane Collaboration [8]. Systematic reviews based on these methods have changed the landscape of health research, but the methods themselves have been criticised as being unable to address questions of great complexity, such as exploring patients' perceptions of disease, identifying underlying theories to explain the effectiveness of an intervention, or understanding the facilitators of and barriers to the uptake of an intervention. Because traditional systematic review methods may be inadequate to address these questions, other types of search approaches (eg, snowballing of papers, focusing on identification of key theories) and analysis techniques (eg, thematic analysis, grounded theory) may be required.

Consider the following questions: "What are the processes involved with bioterrorism preparedness among nurses?" [9] "What is the lived experience of chronic illness among adolescents, and what recommendations can we make for clinical practice regarding that lived experience?" [10] "How can we better understand the dimensions, processes, and practices of 
return to work following illness?" [11]. Such complex questions go beyond a simple consideration of what is effective and have resulted in the emergence of other knowledge synthesis methods. For example, the first question has been approached using concept synthesis [12], the second was answered using critical interpretive synthesis [13], and the third was answered through meta-ethnography [14].

Although emerging knowledge synthesis methods can be fruitful in providing answers for decision-makers, they are challenging to find because they are employed across multiple disciplines, such as philosophy, education, and social science. As well, similar terms are used to describe different methods; for example, each of the terms "meta-ethnography," "metanarrative," and "meta-interpretation" refers to a distinct method. We aimed to identify and chart the various types of emerging knowledge synthesis methods, in terms of their definitions, comprehensiveness, disciplines, and objectives, through a scoping review of studies using emerging synthesis methods. This is the second in a series of articles reporting the results of our scoping review. The first article provides further details about the importance of research on the topic of emerging knowledge synthesis methods and our rationale for undertaking this scoping review [15]. 


\section{Methods}

As outlined in our previously published protocol [16], our scoping review was predicated on the methods outlined by Arksey and O'Malley [17]. We selected a scoping review methodology because it is the most appropriate to address our objectives: to map the literature of evidence that is complex, largely understudied, and dispersed across multi-disciplinary fields, and to identify gaps where primary methods evidence is lacking and needed. As our methods have been presented in detail previously [16], they are described only briefly here.

\subsection{Information sources}

We searched several databases from inception to December 2011: MEDLINE, CINAHL, Embase, PsycINFO, the Cochrane Methodology Register, the Cochrane Database of Systematic Reviews, Social Sciences Abstracts, Library and Information Science Abstracts (LISA), Philosopher's Index, and Education Resources Information Center (ERIC). The literature search was supplemented by scanning the reference lists of included studies, as well as searching textbooks for details about the methods identified. The full literature search strategy for MEDLINE was published previously [16], and the other database search strategies are available upon request.

\subsection{Inclusion criteria}

We included any type of paper, study, report, dissertation, book chapter, or complete book that evaluated, used, or described emerging knowledge synthesis methods in health care. Our definition of these methods was developed with input from experts and was based on the authors' experience. Methods meeting our criteria were those that (1) were not fully operationalised through the use of existing methods manuals and reporting guidelines and (2) were less frequently published in the health care literature than more standard methods. As such, 
122

123

124

125

126

127

128

132

135

136

137

138

139

140

141

142

143

we excluded the following synthesis methods: systematic reviews of treatments and interventions, prognostic reviews, diagnostic reviews of test accuracy, aetiology reviews, economic reviews, and scoping (or mapping) reviews. We included methods from the disciplines of psychology, education, sociology, philosophy, and health as per the World Health Organization's definition [18].

\subsection{Screening and data abstraction}

Two reviewers independently screened the literature search results against the eligibility criteria. Potentially relevant full-text papers were screened in the same manner. Discrepancies were resolved through discussion.

A data abstraction form was created in Excel, and all included studies were independently abstracted by two reviewers. Discrepancies were resolved through discussion. The abstracted data focused on country of conduct (based on corresponding author's address), definitions of the method (according to the study authors), comprehensiveness of the method (ie, whether it could be used for the entire synthesis process - searching for evidence, abstracting data, and analyzing data—or just for the analysis), the discipline on which the paper focused, and the authors' objectives in conducting the study. Disciplines were classified using subject categories in the Web of Science for the journal in which the paper was published. The papers were also classified as an application (or case example) of the method, a review article of multiple methods, or a seminal article that operationalised the steps involved in performing the method. Finally, the studies were classified as to whether they could be used to synthesise qualitative data only or mixed qualitative and quantitative data.

\subsection{Synthesis}


144 We summarised the results descriptively. Qualitative analysis was conducted using

145 NVivo 10 [19] to chart the definitions, comprehensiveness, disciplines, and objectives for the

146 included methods. To depict the frequency of the most common knowledge synthesis methods,

147 we created a word cloud using Wordle [20]. Two reviewers independently identified themes for

148 knowledge synthesis objectives using thematic analysis; discrepancies were resolved through

149 discussion. Methods with substantial overlap in terminology were identified using thematic

150 analysis of the definitions during several team meetings. These methods were grouped together,

151 and the most frequently occurring term was used for referring to the method. As well, similarities

152 between knowledge synthesis methods were deciphered using thematic analysis of the

153 definitions during several team meetings.

154 
155

156

157

158

159

160

161

162

163

164

165

166

167

168

169

170

171

172

173

174

175

176

177

\section{Results}

\subsection{Literature search}

We screened a total of 17,962 titles and abstracts and 1,010 potentially relevant full-text papers and found 409 articles that fulfilled our eligibility criteria (Figure 1; Appendix A and B). Some papers were review articles that described the conduct of multiple emerging knowledge synthesis methods. As such, each article contributed multiple lines of data that were analyzed in NVivo (523 lines of data in total).

\subsection{Characteristics of included articles}

Most of the 409 included articles were published after the year 2000, with $<5 \%$ published before that time (Table 1). The authors of many of the papers were from Europe $(47.2 \%)$ or North America (40.1\%). The methods were used across a wide range of disciplines, with nursing $(33.5 \%)$ and health care sciences and services $(16.1 \%)$ being the most common. The type of article was often an application of the method; $<5 \%$ were seminal articles that described the method in detail (ie, operationalised the steps). The type of evidence most frequently synthesised by the methods was qualitative (70.2\%); the remainder of the studies presented mixed qualitative and quantitative evidence $(29.8 \%)$.

\subsection{Type of knowledge synthesis methods}

Twenty-five unique knowledge synthesis methods were identified. Twelve of these were identified as methods that could be used for the entire knowledge synthesis process (ie, provided guidance on the search strategy, article selection, and analysis), with meta-synthesis being the most common and concept synthesis the least common (Figure 2; Table 2; Appendix C). In contrast, 13 could be used only for the analysis (ie, provided guidance only on the analysis aspect of the knowledge synthesis), with thematic analysis the most common and ecological 
triangulation, inductive analysis, integral meta-theory, philosophic analysis, and pragmatic utility being the least common.

\subsection{Most frequent knowledge synthesis methods by discipline}

Papers using full knowledge synthesis methods were most commonly published in journals within the nursing discipline (31.8\%), and articles that reported using meta-ethnography were published in the widest variety of disciplines relative to any of the other methods used for full knowledge synthesis (Table 3, Appendix D and E). Papers using analysis methods were also most commonly published in journals in the fields of nursing (7.6\%) and articles that reported using thematic analysis were published in the widest variety of disciplines relative to any of the other analysis methods.

\subsection{Most frequent objectives for knowledge synthesis methods}

Using thematic analysis, we identified 11 main objectives across the included articles, which are outlined in Appendix F. Among the full knowledge synthesis methods, the most common objective was to explore a phenomenon (38.4\%). Among studies that reported analysis methods, the most common objective was to explore different knowledge synthesis methods $(13.7 \%)$.

\subsection{Overlap in terminology among knowledge synthesis methods}

According to thematic analysis of the definitions, we found overlap in the terminology used to describe the knowledge synthesis methods. Methods with overlapping terminology were grouped, and the most frequent term was used to describe the method. For full knowledge synthesis methods, synonymy of terminology is indicated in Table 2. Critical interpretive synthesis was the method with the highest number of overlapping terms (critical literature review, critical review, interpretive approach, interpretive synthesis, and meta-interpretation). 
For two of the full knowledge synthesis methods, meta-interpretation and meta-study, there were no synonyms.

The findings were similar for the analysis methods. For the analysis methods, synonymy of terminology is indicated in Appendix C. Thematic analysis had the highest number of synonymous terms methods, including thematic framework, thematic literature review, thematic review, thematic synthesis, thematic content analysis, thematic meta-analysis, and comparative thematic approach. Overlap in terminology was not observed for content analysis, ecological triangulation, inductive analysis, integral meta-theory, philosophic analysis, and pragmatic utility.

\subsection{Similarities among full knowledge synthesis methods}

Using thematic analysis, we identified two main groupings of the full knowledge synthesis methods: methods that can be used to integrate qualitative and quantitative data and methods that can be used to establish or refine a theory, perspective, or phenomenon (Figure 3). Methods exclusively for integrating qualitative and quantitative data were meta-summary, metanarrative review, and mixed studies reviews. Methods exclusively for establishing a new theory, perspective, or phenomenon were concept synthesis, meta-ethnography, meta-interpretation, meta-study, and meta-synthesis. Four methods can be used for both functions: critical interpretive synthesis, integrative review, realist review, and narrative synthesis. 


\section{Discussion}

Through our comprehensive scoping review, we identified 25 unique knowledge synthesis methods across 409 articles. About half of these reports described full knowledge synthesis methods whereas the remainder described methods that could be used only for analysis. Most of the methods were contemporary and likely emerging; indeed, $81.1 \%$ of the included articles were published after the year 2005. These 25 methods were most commonly used across journals in the fields of nursing, health care science and services, and health policy. Finally, we observed numerous applications of the various methods across the literature, yet we identified few seminal articles that operationalised the steps of these methods. This finding suggests that further empirical work to formalise these methods is required.

These results can be used to advance the knowledge synthesis field. We have provided a

231 framework of terminology and definitions for the 25 knowledge synthesis methods. These methods will likely be useful to answer complex questions that go beyond consideration of what is effective, for example, "How do young people (aged 13-19 years) with epilepsy experience information exchange in health care contexts, and what factors influence positive and negative health care communication?" [21] and "How can we use a policy assessment tool to evaluate the outcomes of policies promoting increased accessibility to services in rural areas?" [22]. Using thematic analysis, we grouped the 79 individual methods identified as 25 unique methods. During this exercise, we aimed not to be overly reductionist. For example, narrative

239 reviews and meta-narrative reviews have some similar aspects, because both begin with

240 preliminary synthesis of findings through identification of key theories and because both aim to 241 narratively describe the literature. However, we kept these methods separate because slightly 242 different terminology was used and the methods seemed to differ slightly. Among the analysis 
methods, techniques from content analysis are used in the case survey method, aggregative analysis, and inductive analysis, yet we kept these methods separate as well, again because of differing terminology. These issues will be further examined during a proposed meeting of experts in the field, described in more detail below.

Our results may also be helpful because we identified many full knowledge synthesis methods, along with the types of review objectives that can be met by the various approaches. For example, a researcher who wishes to explore a phenomenon could consider content analysis, framework analysis, grounded theory, or thematic analysis. Alternatively, to explore perceptions, meta-summary, meta-synthesis, and mixed studies reviews might be of use.

To further group methods and develop an algorithm for matching the most appropriate knowledge synthesis method to any knowledge synthesis question (or objective), our next step will be to convene a meeting of an international group of leaders in the field. We anticipate that this group will further clarify the emerging knowledge synthesis approaches identified here. For example, subgroups can answer the same research question using different knowledge synthesis methods and results from the various synthesis approaches will be compared to determine whether the answers differ according to the method used. We anticipate that this exercise will refine the methods identified in our scoping review. We encourage readers who are interested in joining this international group to contact the corresponding author.

Although our scoping review was comprehensive, it had some limitations. Like all scoping reviews, ours involved no assessment of risk of bias or meta-analysis, and its focus was on providing breadth rather than depth about a particular topic [23]. However, this approach was appropriate, given our objective of mapping out the literature on a largely understudied and complex topic. Additionally, we applied rigorous methods (including a calibration process for 
study selection, duplicate screening, and use of standardised data abstraction), and we adhered to current standards for the conduct of scoping reviews, as outlined by Arksey and O'Malley [17].

We did not comprehensively search the grey literature beyond conference abstracts, protocols, and dissertations. Also, to increase the efficiency of our review, we limited our coverage to articles written in English. Although we searched the literature outside health databases, our search focused predominantly on health-related literature across the areas of psychology, education, sociology, and philosophy. As a consequence, our results likely reflect the English literature in health databases, and we may have missed some important knowledge synthesis methods written in other languages and applied in disciplines other than health. Furthermore, it was difficult to classify the 79 methods initially identified into 25 unique methods, and there may have been some misclassification. Additionally, we have reported our findings according to two distinct features of full knowledge synthesis methods that emerged from our data: methodological activity (ie, integrating quantitative and qualitative evidence) and purpose (ie, generate theory), but there may be alternative ways to represent the differences among different knowledge synthesis methods. However, we expect that any false assumptions or misclassifications will be resolved during our planned international meeting of experts. Finally, because of the sheer size of this scoping review, we were unable to include other important work published later than our search date, such as textbooks published by Gough and colleagues [24], Pawson and colleagues [25], and Hannes and colleagues [26]. However, these textbooks will be considered in the development of an algorithm during the international meeting. and limitations, and key knowledge and skills required for conducting full knowledge synthesis 
288 methods in the two main groupings, those for integrating qualitative and quantitative data [27]

289 and those for establishing or refining a theory, perception, or phenomenon [28].

290

$-16-$ 
291 FIGURE LEGENDS

292 Figure 1: Study flow diagram

293 Figure 2: Word cloud for most frequent knowledge synthesis methods

294 Figure 3: Similarities between the full knowledge synthesis methods 295 


\section{Competing interests}

297 The authors have no competing interests to declare.

\section{Acknowledgements}

299 The study was funded by a Canadian Institutes of Health Research (CIHR) Knowledge Synthesis 300 grant. ACT holds a CIHR/Drug Safety and Effectiveness Network New Investigator Award in

301 Knowledge Synthesis, and SES holds a Tier 1 Canada Research Chair in Knowledge Translation.

302 We thank Drs. Jeremy Grimshaw, David Moher, and Peter Tugwell, who provided support and

303 expertise in knowledge synthesis methods and knowledge translation for our scoping review

304 protocol.

\section{Authors' contributions}

306 ACT conceived the study, obtained funding for the study, screened citations and full-text articles,

307 abstracted data, analyzed and interpreted the data, and wrote the manuscript. CS and JA

308 coordinated the study, screened citations and full-text articles, abstracted data, developed the

309 qualitative analysis, coded and analyzed data, and edited the manuscript. EC screened citations

310 and full-text articles, abstracted data, and edited the manuscript. HM and EL screened citations

311 and full-text articles, abstracted and cleaned data, and edited the manuscript. JT coded data in

312 NVivo, generated tables and figures, and edited the manuscript. JD and WH coded data in NVivo

313 and edited the manuscript. LP developed the literature search, screened citations and full-text

314 articles, abstracted and cleaned data, and edited the manuscript. VW screened citations and full-

315 text articles, interpreted the results, and edited the manuscript. TH interpreted the results and

316 edited the manuscript. SES conceived the study, obtained funding for the study, analyzed and

317 interpreted the data, and edited the manuscript. MK conceived the study, obtained funding for 
318 the study, screened citations and full-text articles, abstracted data, analyzed and interpreted the 319 data, and edited the manuscript. 
Table 1: Summary of papers included in the scoping review

Characteristic

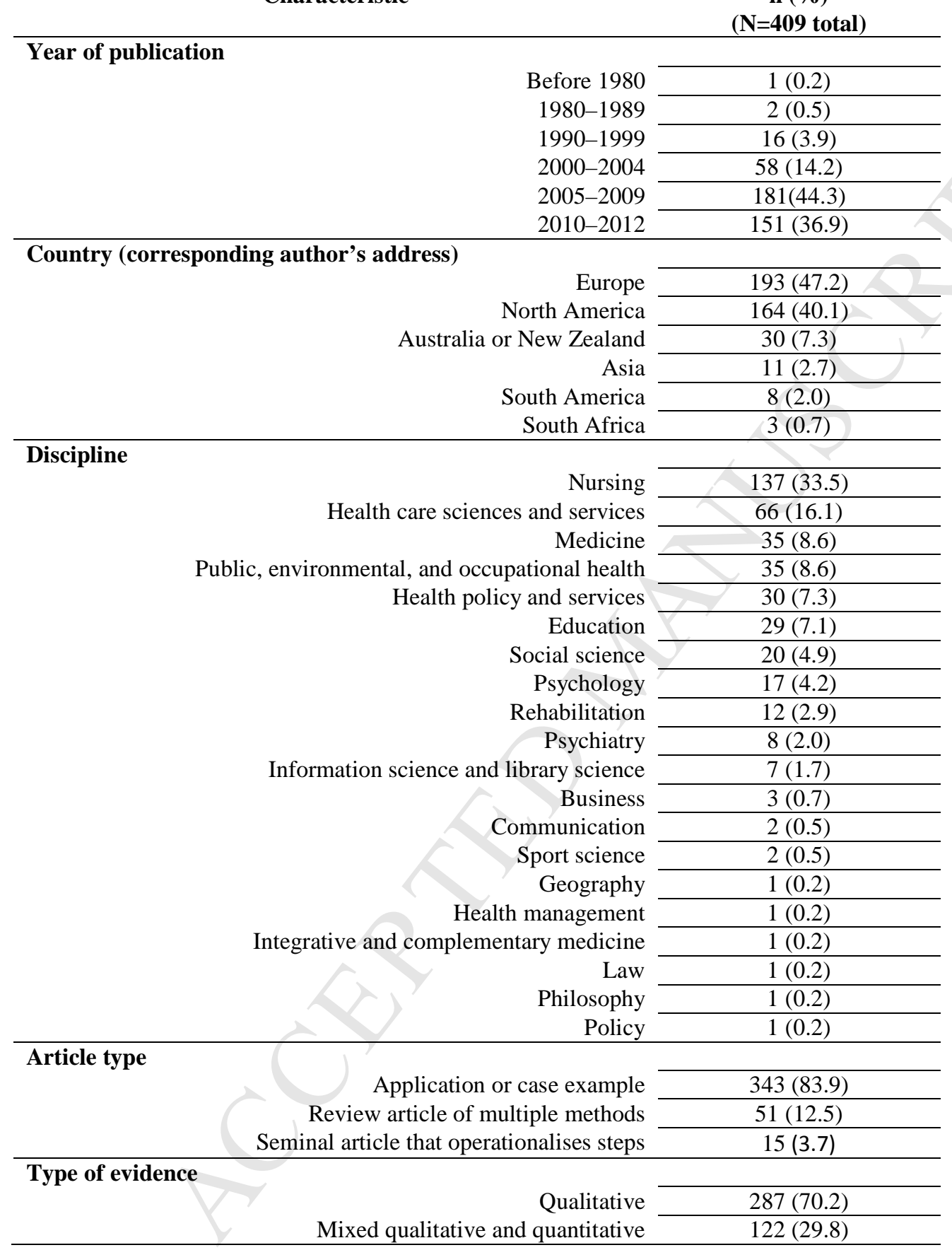

$\mathrm{N}=409$ total

Before 1980

1980-1989

2000-2004

005-2009

$10-2012$

Europe aland

\section{Articles,}

n (\%) 
322 Table 2: Frequency and definitions of the full knowledge synthesis (KS) methods

\section{KS Method (N)*; Method(s) with similar definitions}

Integrative review (36); Integrative literature review, integrative review with conceptual framework Meta-synthesis (95); Qualitative meta-synthesis

Mixed studies review (15); Mixed methods approach, mixed methods review, mixed methods synthesis, mixed methods systematic review

Meta-interpretation (7); None identified

Unbrella term used to describe synthesis methods for integrating qualitative and quantitative data. Can be used to draw conclusions that provide a more comprehensive understanding of a particular phenomenon than that existing before the review.

Concept synthesis (7); Conceptual framework synthesis model, conceptual model development, conceptual review, conceptual analysis Critical interpretive synthesis (17); Critical literature review, critical review, interpretive approach, interpretive synthesis, metainterpretation

Meta-ethnography (61);

Extended meta-ethnography Umbrella term used to combine separate elements to form a coherent whole using a process of logical deduction. It aims to provide an accurate interpretation of a phenomenon and to compare and contrast individual studies to reach consensus on a new understanding of that phenomenon. It is an integrative and interpretive method that allows themes or key metaphors to emerge. Umbrella term used to describe reviews combining or integrating (i) qualitative and quantitative studies, (ii) only mixed methods studies, or (iii) mixed methods studies and either qualitative or quantitative studies (or both).

Umbrella term used to describe theoretical synthesis methods that provide a broader understanding of human behaviour and experience and should lead to new insights that are not observed in the original studies. It is an iterative process that uses interpretation of interpretations and theoretical sampling of studies for synthesis until theoretical saturation is reached. Synthesis method used to identify concepts, viewpoints or ideas. Focuses on identifying the defining attributes of the concepts and can be used to develop a synthesis model.

Synthesis method that uses an iterative approach to refine the research question, search and select articles from the literature, and define and apply codes and categories. Data is re-categorised and interpreted, which results in a comprehensive critical narrative using qualitative and quantitative evidence that is grounded in data reported in the included studies. May result in new typologies, concepts, models or theory. Adopted from qualitative research areas (eg, ethnography, historical research and hermeneutics).

Technique to synthesise qualitative research, or develop "translations of qualitative studies into one another" (ie, reciprocal translation analysis). Interpretive approach that aims to provide a new interpretation of these studies or a new theory to explain the range of research findings encountered, rather than a simple aggregation. A way of re-analyzing and comparing the texts of published studies (rather than the original data of each) to produce a new interpretation. Involves induction and interpretation, whereby separate parts are brought together to form a "whole" (ie, looking for a new theory or 'line of argument' to explain all the studies) so that the result is greater than the sum of its parts.

Meta-narrative review (6); Method developed from the need to synthesise evidence to inform complex policy-making questions. Involves looking across Meta-narrative, meta-narrative different paradigms or research traditions to uncover their "unfolding storyline," which results in maps of "meta-narratives" from mapping analysis which dimensions or themes can be revealed and distilled. Useful for exploring differences between research traditions and making sense of conflicting results.

Meta-study (27)

None identified

A multifaceted, interpretive approach to synthesis developed to study the experiences of patients living with chronic illness. Consists of three components to be completed before synthesis: meta-data-analysis, meta-method and meta-theory. Collectively, these create a new interpretation accounting for the results of all three elements of analysis (called meta-synthesis).

Meta-summary (10); Meta-aggregation, meta-review, meta-summaries, qualitative meta-summary A quantitatively oriented summary of qualitative findings developed to accommodate the distinctive features of qualitative surveys. Can be used to combine descriptive quantitative and qualitative studies. The approach includes the extraction, grouping and formatting of findings, and the calculation of frequency and intensity effect sizes, which can be used to produce mixed research syntheses and to conduct analyses of the relationship between reports and findings. May be used to develop a map of qualitative studies, which can serve as a basis for a further synthesis.

Narrative synthesis (10);

Narrative and theoretical syntheses, narrative review, narrative summary Synthesis method that includes a formal analytical process to generate new insights or knowledge by seeking to be systematic and transparent. Involves the juxtaposition of findings from the studies included in the review and some element of integration or interpretation. Slight differences in the field have been noted for narrative synthesis and narrative analysis, as described below: Narrative synthesis

Draws out central theories or causal mechanisms identified in multiple studies and builds an explanation of the body of research by telling the story of the evolution of the field or mapping the domains covered by the literature in an area. Created using the methods of thematic analysis, conceptual mapping and critical reflection on the synthesis process. Process of compiling descriptive data and exemplars from individual studies and building them into a mosaic or map (ie,meta-narrative mapping). Narrative review (qualitative)

Qualitative systematic review that uses a narrative inductive method of analysis. Allows for qualitative description, interpretation and synthesis, leading to identification of common and emergent themes, rather than testing theory or evaluating study findings.

Realist review (27);

Realist synthesis A method rooted in realist philosophy that is used to investigate "what works for whom, under what circumstances, and why."

Primary focus is on the causal mechanisms or "theories" that underlie types of interventions or programs, aiming to build explanations across interventions or programs that share similar underlying "theories of change" as to why they work (or do not work) for particular groups in particular contexts.

323 Note: *References for each method are shown in Appendix A. 


\section{Table 3: Methods by type of evidence, discipline and objective for the full knowledge} 325 synthesis (KS) methods according to authors of articles describing the method

\begin{tabular}{ll}
\hline \multicolumn{1}{c}{ KS Method* } & Type of evidence used \\
Integrative & $\begin{array}{l}\text { Qualitative data and } \\
\text { quantitative data }\end{array}$ \\
\hline $\begin{array}{l}\text { Mixed studies } \\
\text { review }\end{array}$ & $\begin{array}{l}\text { Qualitative data and } \\
\text { quantitative data and } \\
\text { mixed data }\end{array}$ \\
\hline
\end{tabular}
quantitative data

Realist review Qualitative data and

\section{Discipline*}

Education; Health care services and

science; Health policy and services;

Medicine; Nursing; Public,

environmental, and occupational health

Education; Health care services and

science; Health policy and services;

Information science and library science;

Medicine; Nursing; Public,

environmental, and occupational health;

Social science

Business; Education; Health care

services and science; Health

management; Health policy; Integrative

and complementary medicine; Law;

Medicine; Nursing; Public,

environmental, and occupational health; Rehabilitation; Social science

\begin{tabular}{ll}
\hline Critical & Qualitative data and \\
interpretive & quantitative data; \\
synthesis & qualitative data alone
\end{tabular}

Education; Health care services and science; Health policy and services; Information science and library science; Nursing; Psychiatry; Rehabilitation; Social science

\begin{tabular}{ll}
\hline $\begin{array}{l}\text { Narrative } \\
\text { synthesis }\end{array}$ & $\begin{array}{l}\text { Qualitative data and } \\
\text { quantitative data; } \\
\text { qualitative data alone }\end{array}$ \\
\hline $\begin{array}{l}\text { Meta-narrative } \\
\text { review }\end{array}$ & $\begin{array}{l}\text { Qualitative data and } \\
\text { quantitative data; }\end{array}$ \\
& Qualitative data alone
\end{tabular}

Qualitative data alone

\begin{tabular}{ll}
\hline Meta-summary & $\begin{array}{l}\text { Qualitative data and } \\
\text { quantitative data; }\end{array}$
\end{tabular}

Qualitative data alone

Meta-synthesis Qualitative data

Business; Health care services and science; Health policy and services; Nursing; Psychology

Health care services and science; Health policy and services; Medicine; Public, environmental, and occupational health; Social science

Health care services and science; Health policy and services; Medicine; Nursing; Public, environmental, and occupational health; Social science

Education; Health care services and science; Health policy and services; Information science and library science; Medicine; Nursing; Psychiatry; Psychology; Public, environmental, and occupational health; Rehabilitation; Social science

Meta- Qualitative data
Business; Communications; Education;

ethnography Geography; Health care services and science; Health policy and services; Information science and library science Medicine; Nursing; Psychiatry; Psychology; Public, environmental, and occupational health; Rehabilitation; Social science; Sport science

\begin{tabular}{ll}
\hline $\begin{array}{l}\text { Meta- } \\
\text { interpretation }\end{array}$ & Qualitative data \\
& \\
\hline Concept & Qualitative data
\end{tabular}
Health care services and science; Health policy and services; Nursing; Public, environmental, and occupational health; Social science; Sport science

\begin{tabular}{|c|c|c|}
\hline $\begin{array}{l}\text { Concept } \\
\text { synthesis }\end{array}$ & Qualitative data & $\begin{array}{l}\text { Information science and library science } \\
\text { Medicine; Nursing; Psychiatry; } \\
\text { Rehabilitation }\end{array}$ \\
\hline Meta-study & Qualitative data & $\begin{array}{l}\text { Education; Geography; Health care } \\
\text { services and science; Health policy and } \\
\text { services; Nursing; Psychology; Public, } \\
\text { environmental, and occupational health; } \\
\text { Rehabilitation; Sport science }\end{array}$ \\
\hline
\end{tabular}
Explore understanding

Explore perceptions Explore a phenomenon Review and evaluate Explore perceptions Explore a phenomenon Explore synthesis methods

\section{Categories of objectives $\dot{\dagger}$}

Explore synthesis methods

Explore qualitative research

Develop/describe framework

Investigate barriers and facilitators
Explore understanding

Explore a phenomenon

Review and evaluate

Explore synthesis methods

Develop/describe frameworks

Study policy and decision making

Tool development

Explore understanding

Explore perceptions

Explore a phenomenon

Study process and relationships

Explore synthesis methods

Explore perceptions $\quad$ Explore synthesis methods

Explore a phenomenon $\quad$ Develop/describe frameworks

Review and evaluate

Explore understanding Explore qualitative research

Explore a phenomenon $\quad$ Develop/describe frameworks

Review and evaluate

Explore synthesis methods

Explore perceptions

Explore a phenomenon

Explore synthesis methods

Explore qualitative research

Explore understanding

Explore perceptions

Explore a phenomenon

Review and evaluate

Study process and relationships

Explore synthesis methods

Explore qualitative research

Explore understanding

Explore perceptions

Explore a phenomenon

Review and evaluate

Study process and relationships

Explore synthesis methods

Explore qualitative research

Develop/describe frameworks

Explore perceptions

Explore a phenomenon

Explore synthesis methods

\section{Explore understanding}

Explore a phenomenon

Explore synthesis methods

Explore understanding

Explore perceptions

Explore a phenomenon

Review and evaluate

Explore synthesis methods 326 Note: *Categorised according to Web of Science classification. $†$ Develop/describe frameworks also includes guidelines, models, measures/scales, people perceive and experience a phenomenon, disease or health state; Review and evaluate = aim is to broadly synthesise the literature on a health topic, including the effectiveness of an intervention.

330 Abbreviation: KS, knowledge synthesis 


\section{References}

[1] O'Rourke K. An historical perspective on meta-analysis: dealing quantitatively with varying study results. Journal of the Royal Society of Medicine. 2007;100:579-82.

[2] Report on Certain Enteric Fever Inoculation Statistics. British medical journal. 1904;2:12436.

[3] Glass GV. Primary, Secondary, and Meta-Analysis of Research. Educational Researcher American Educational Research Association. 1976;5:3-8.

[4] Slavin RE. Best-Evidence Synthesis: An Alternative to Meta-Analytic and Traditional Reviews. Researcher. Educational Researcher American Educational Rsearch Association. 1986;15:5-9.

[5] Glass GV, McGaw B, Smith ML. Meta-Analysis in Social Research (Sage Library of Social Research). Beverly Hills: Sage Publications; 1981. p. 279.

[6] Rosenthal R. Combining results of independent studies. Psychological Bulletin. 1978;85:18593.

[7] Cooper HM, R.A. R. Comparison of Statistical and Traditional Procedures for Summarizing Research. Psycological Bulletin. 1980;87:442-9.

[8] Higgins J, Green S, (editors). Cochrane Handbook for Systematic Reviews of Interventions Version 5.1.0 [updated March 2011]. The Cochrane Collaboration, 2011. Available from www.cochrane-handbook.org.

[9] Rebmann T. Nursing bioterrorism preparedness [Dissertation]. Saint Louis: Saint Louis University; 2006.

[10] Taylor RM, Gibson F, Franck LS. The experience of living with a chronic illness during adolescence: a critical review of the literature. Journal of clinical nursing. 2008;17:3083-91. [11] MacEachen E, Clarke J, Franche RL, Irvin E, Workplace-based Return to Work Literature Review G. Systematic review of the qualitative literature on return to work after injury. Scandinavian journal of work, environment \& health. 2006;32:257-69.

[12] Walker LO, Coalson K. Strategies for Theory Construction in Nursing. 4th ed: Prentice Hall; 2005.

[13] Dixon-Woods M, Cavers D, Agarwal S, Annandale E, Arthur A, Harvey J, et al. Conducting a critical interpretive synthesis of the literature on access to healthcare by vulnerable groups. BMC medical research methodology. 2006;6:35.

[14] Noblit GW, Hare RD. Meta-ethnography. Newbury Park, CA.: SAGE Research Methods; 1988.

[15] Straus SE, Kastner M, Soobiah C, Antony J, Tricco AC. Engaging researchers on developing, using and improving knowledge synthesis methods: an introduction to results of a scoping review . In press at the Journal of Clinical Epidemiology; Manuscript No JCE-14-701, 8 pages.

[16] Kastner M, Tricco AC, Soobiah C, Lillie E, Perrier L, Horsley T, et al. What is the most appropriate knowledge synthesis method to conduct a review? Protocol for a scoping review. BMC medical research methodology. 2012;12:114.

[17] Arksey H, O'Malley L. Scoping studies: Towards a Methodological Framework. International Journal of Social Research Methodology. 2005;8:19-32.

[18] WHO. Preamble to the Constitution of the World Health Organization as adopted by the International Health Conference, New York, 19-22 June, 1946; signed on 22 July 1946 by the 
representatives of 61 States (Official Records of the World Health Organization, no. 2, p. 100) and entered into force on 7 April 1948. 1946.

[19] International Q. NVivo10 for Windows. 2014.

[20] Feinberg J. Wordle. 2013.

[21] Lewis SA, Noyes J, Mackereth S. Knowledge and information needs of young people with epilepsy and their parents: Mixed-method systematic review. BMC pediatrics. 2010;10:103. [22] Bristow GF, J.; Shaw, J.; Richardson, T. Developing an evaluation framework for crosscutting policy goals: the Accessibility Policy Assessment Tool. Environment and Planning A. 2009;41:48-62.

[23] Tricco A, Lillie E, Zarin W, O'Brien K, Colquhoun H, Kastner M, et al. Conduct and quality of reporting of scoping reviews: A scoping review. Under review at Implementation Science. 2015. [24] Gough D, Oliver S, Thomas J. An introduction to Systematic Reviews.: Sage Publications; 2012.

[25] Pawson R. The Science of Evaluation: A Realist Manifesto: Sage Publications Inc.; 2013. [26] Hannes K, Lockwood C. Synthesizing Qualitative Research: Choosing the right approach. : Wiley-Blackwell \& Sons Ltd.; 2012.

[27] Tricco AC, Antony J, Soobiah C, Kastner M, MacDonald H, Cogo E, et al. Knowledge synthesis methods for integrating qualitative and quantitative data: A scoping review reveals poor operationalisation of the methodological steps. In press at the Journal of Clinical Epidemiology; Manuscript number: JCE-14-896.

[28] Tricco AC, Antony J, Soobiah C, Kastner M, Cogo E, MacDonald H, et al. Knowledge synthesis methods for generating or refining theory: A scoping review reveals that little guidance is available. In press at the Journal of Clinical Epidemiology; Manuscript No: JCE-15-43R1. 
$\mathrm{N}=17,962$ titles and abstracts from MEDLINE, CINAHL, ERIC, LISA and other sources

\begin{tabular}{|l|l|l|}
$\begin{array}{l}\mathrm{N}=16,952 \text { excluded } \\
\text { 1) }\end{array}$ & $\begin{array}{l}\text { Not a knowledge synthesis method } \\
(\mathrm{n}=16,915)\end{array}$ \\
$2)$ & $\begin{array}{l}\text { Not related to health or philosophy } \\
(\mathrm{n}=37)\end{array}$
\end{tabular}

$\mathrm{N}=1,010$ potentially relevant full-text articles

$\mathrm{N}=601$ excluded

1) Not a knowledge synthesis method $(n=457)$

2) Non-English study $(n=72)$

3) Unable to locate full text $(n=69)$

4) Not related to health or philosophy $(n=3)$

$\mathrm{N}=409$ included articles reporting on 25 methods 


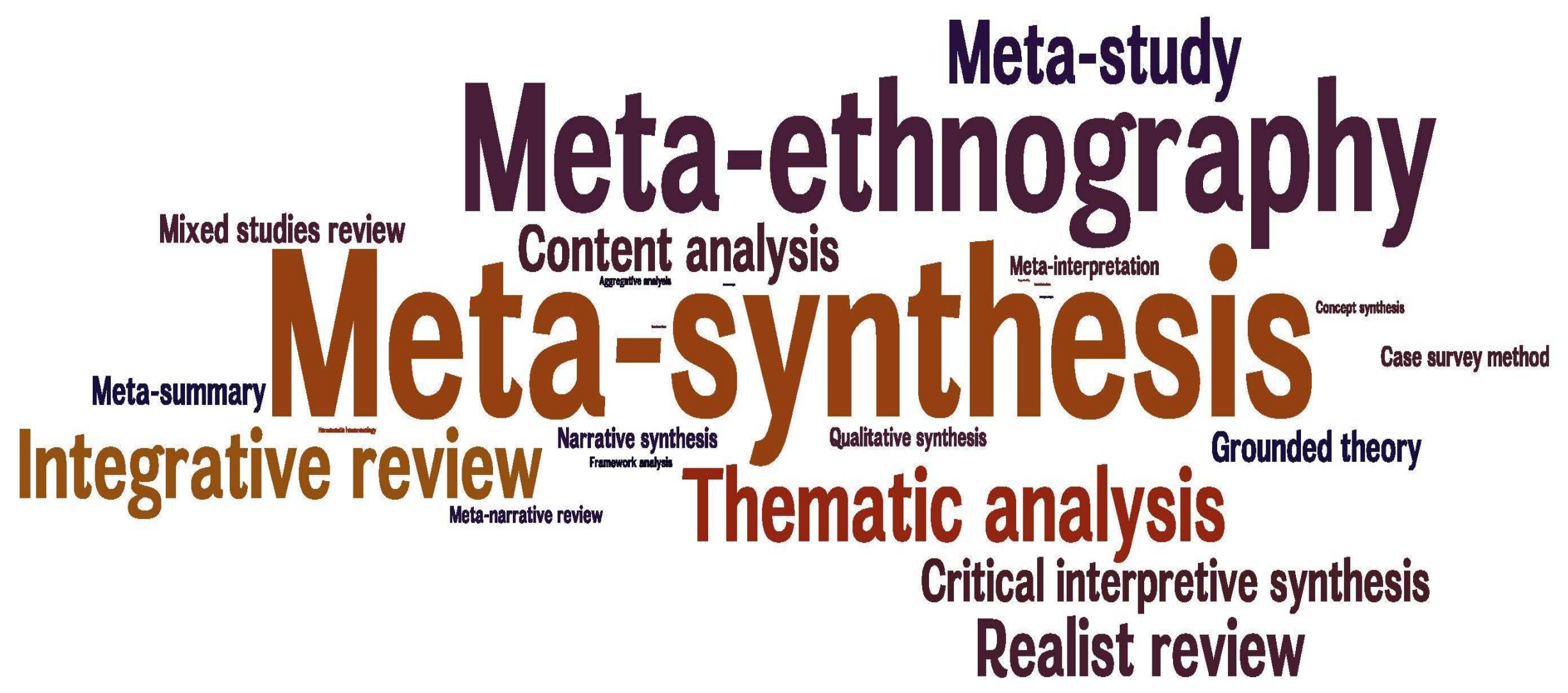


Integrate qualitative and quantitative studies
Establish or refine a theory, perspective or phenomena

Concept synthesis

Meta-summary Critical interpretive synthesis

Meta-narrative review Integrative review Th:

Mixed studies review Realist review

Narrative synthesis

Meta-ethnography

Meta-interpretation

Meta-study

Meta-synthesis 